\title{
Use of heavy Trypan blue in macular hole surgery
}

\begin{abstract}
Purpose Intraocular dyes facilitate the identification of the inner limiting membrane (ILM) during surgery. Appropriate dyes should be safe, provide adequate staining, and be easy to use. Heavy Trypan blue eliminates the need for an air-fluid exchange (AFX) and appears to have little retinal toxicity. This study refers to a prospective, consecutive trial with heavy Trypan blue in macular hole surgery.

Patients and methods A consecutive group of 20 patients with full thickness macular holes was recruited in a single institution study. Patients were operated using conventional methods. Heavy Trypan blue was prepared by mixing isovolumetrically glucose $10 \%$ with MembraneBlue (DORC, Zuidland, The Netherlands). Patients were assessed for ease of surgery and post-operatively at 3 and 6 months (vision and ocular coherence tomography) for hole closure and vision.

Results Twenty eyes were included in the study. Reapplication of dye was used in $75 \%$ of the cases, leading to improved contrast further facilitating the ILM peel. In no case was an AFX necessary to obtain sufficient staining. Macular hole closure was achieved in 19 of 20 patients with one surgery. No retinal detachment or other complication was observed in the follow-up period.

Conclusion Heavy Trypan blue can be delivered efficiently to the retinal surface without the need for an AFX. Staining was sufficient to help visualise and peel the ILM. Repeat applications were easily performed. The macular hole closure rate was similar to that of other series with a comparable visual improvement.

Eye (2010) 24, 1177-1181; doi:10.1038/eye.2010.3; published online 12 February 2010
\end{abstract}

Keywords: Trypan blue; staining; macular hole; inner limiting membrane; vitreoretinal surgery

SY Lesnik Oberstein ${ }^{1}$ and MD de Smet ${ }^{1,2}$

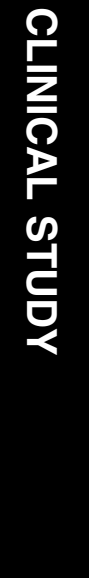

\section{Introduction}

Removal of inner limiting membranes (ILM) and idiopathic epiretinal membranes (ERMs) requires skill and experience.

Non-closure of macular holes and re-proliferation of macular puckers have both been related to inadequate membrane removal. To facilitate surgery and improve the surgical outcome, adjuncts to enhance membrane visibility have been sought. These have included the use of a slit beam illumination, triamcinolone, ${ }^{1}$ and stains such as Indocyanine and infracyanine green (ICG), brilliant blue, ${ }^{2,3}$ and Trypan blue. ${ }^{4-8}$ Owing to a superior safety profile as compared with ICG, Trypan blue has been used by a number of European surgeons despite its lesser staining characteristics for the ILM. ${ }^{7,9,10}$

The commercially available version of Trypan blue (MembraneBlue, DORC, Zuidland, The Netherlands) used in vitreoretinal surgery is diluted in phosphate-buffered saline. It requires a fluid-air exchange for the dye to pool over the macular surface as it otherwise diffuses throughout the vitreous cavity, as MembraneBlue has a similar osmolarity to balanced salt solution (BSS) and thus cannot stain membranes sufficiently. The methods to avoid the dispersion of the dye would be to either lower the osmolarity of the infusion solution, such as using Hartmann solution, or by increasing the osmolarity of the dye. In this study, to avoid the need for a fluid-air exchange, we modified the diluents used with Trypan blue to render it denser than saline, $\mathrm{BSS}+$, and other vitreoretinal infusates. This solution requires the addition of a fixed volume of concentrated glucose solution to achieve the necessary density. We used MembraneBlue (DORC) supplied in $0.5 \mathrm{ml}$ of phosphatebuffered saline and mixed it with $0.5 \mathrm{ml}$ of glucose $10 \%$. We have earlier shown the use of heavy Trypan blue to be useful in the peeling of ERMs, achieving good anatomical and functional results. ${ }^{11}$
${ }^{1}$ Department of Ophthalmology, Academic Medical Center, University of Amsterdam, Amsterdam, The Netherlands

${ }^{2}$ Retina and Ocular Inflammation Unit, Center for Specialized Ophthalmology,

Clinique de Montchoisi, Ch des Allinges 10, Lausanne 1001, Switzerland

Correspondence: SY Lesnik Oberstein, Department of Ophthalmology, University of Amsterdam, Rm D2-431, Meibergdreef 9, Amsterdam 1105AZ, The Netherlands. Tel: + 3120566 9111; Fax: + 31205669053 E-mail: S.Y.LesnikOberstein@ amc.uva.nl

Received: 11 June 2009 Accepted in revised form: 26 November 2009 Published online: 12 February 2010 
We now propose using this modified 'heavy' Trypan blue solution in macular hole surgery with ILM peeling. We conducted a prospective, consecutive study of 20 patients with macular holes to assess the staining characteristics, ease of use, and safety of the heavy Trypan blue in macular hole surgery.

\section{Materials and methods}

Patients with idiopathic macular holes were recruited prospectively and consecutively into this study. Pre-operative assessment included age, gender, best-corrected visual acuity (BCVA), and full ocular examination. The primary outcome measure was the staining characteristic of the dye, specifically the number of repeat applications needed to complete the ILM peel. Secondary outcome measures were hole closure rate, post-operative visual acuity, and surgical complications. Ocular coherence tomography (OCT) was performed using the OCT3 (Zeiss Inc., Jena, Germany) to measure the smallest diameter of the macular hole opening, assess closure, and analyse the presence of ERM on the retina. The OCT was obtained by a trained technician using the fast macular thickness protocol. Post-operatively, BCVA and OCT were repeated at 3 and 6 months and hole closure was assessed.

Patients were operated using conventional methods using a 3 port, 20 gauge vitrectomy. After complete vitrectomy, the infusion line was turned off and heavy Trypan blue was injected gently, close over the macular area using a lacrimal cannula, and left for $1 \mathrm{~min}$ (Figure 1a). Heavy Trypan blue was prepared by mixing equal volumes of glucose $10 \%$ with commercially available Trypan blue $0.15 \%$ (MembraneBlue, DORC). The glucose $10 \%$ and MembraneBlue mix easily at room temperature. This provides a final glucose concentration of 5\% and a final Trypan blue concentration of $0.075 \%$.

This solution had an osmolarity of 320 mOsm and a $\mathrm{pH}$ of 7.0, as tested in our laboratory and by the hospital pharmacy. After a 1-min incubation, the infusion was once again opened and the excess dye was removed using a silicone tipped Charles flute (Figure 1a).

Membrane peeling was achieved using a combination of a pic needle or bent MVR blade and Eckardt micro forceps (DORC) (Figure 1b). The staining procedure was repeated in some patients when the ILM stain was not sufficiently intense, or the edge of the ILM was lost, to allow a complete and controlled peel. After full removal of the ILM, an internal search was performed and the sclerotomies and conjunctiva were closed.

We certify that all applicable institutional and governmental regulations concerning the ethical use of human volunteers were followed during this research. Appropriate approval from the hospital medical ethics
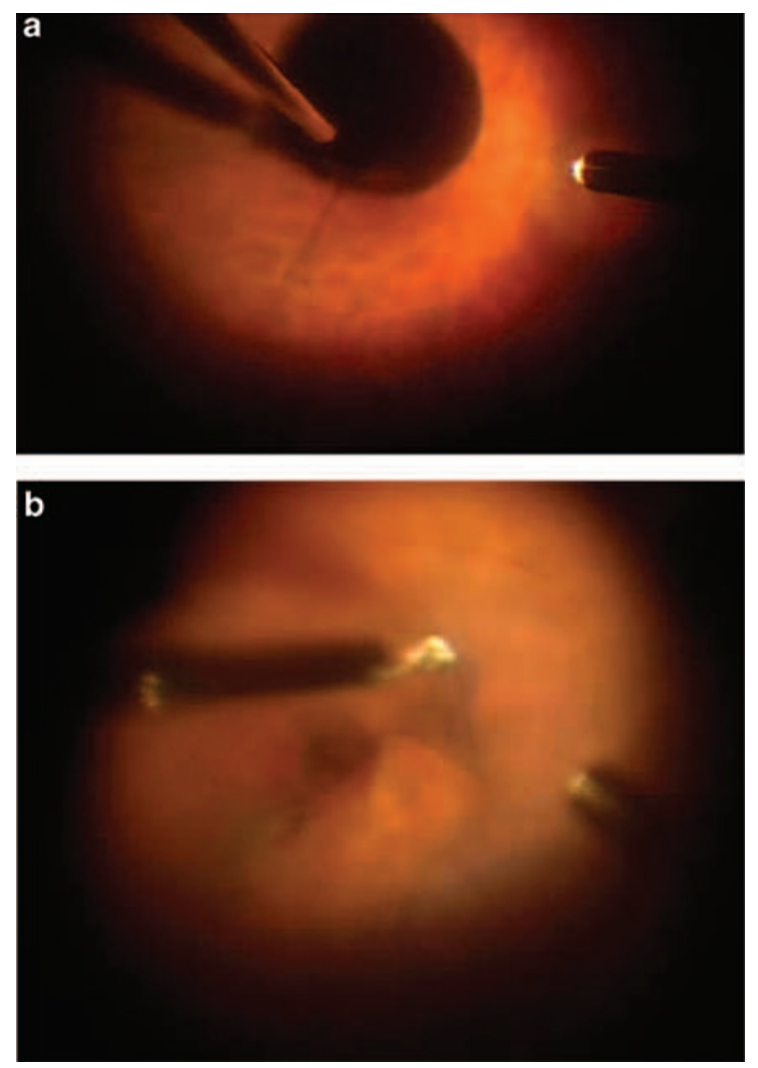

Figure 1 (a) Heavy membrane blue being injected over the macular area. (b) ILM removal after 1 min stain with heavy membrane blue.

committee was obtained as dictated by Dutch law for this type of medical investigation. Appropriate consent was obtained.

\section{Results}

A total of 20 eyes of 20 patients were included in this study. Mean age was 67.5 years (range 54-86). Nine patients were female and 11 male. Four patients were phakic after the 6-month follow-up period and the other 16 had either combined phaco-vitrectomy surgery or were pseudophakic pre-operatively. Sixteen of the macular holes were idiopathic including two eyes of diabetic patients that showed no signs of retinopathy. Two patients were high myopes $(-20$ and $-8 \mathrm{D})$. One patient had an earlier retinal detachment. Pre-operative BCVA in logMAR units ranged from 1.0 to 0.4 (mean 0.7, median 0.8). OCT showed a classic macular hole in all patients. Pre-operatively, 14 patients had a diameter of $<500 \mu \mathrm{m}$ and 6 had a diameter of $500 \mu \mathrm{m}$ or larger. Nineteen of 20 holes closed after one surgery. In 15 of 20 surgeries, heavy Trypan blue dye was reapplied to improve visualisation of the ILM's edge. More than two dye applications were not required in any surgery. 
Table 1 Patient demographics and results

\begin{tabular}{|c|c|c|c|c|c|c|c|c|c|c|c|}
\hline & Age & Sex & Eye & $\begin{array}{l}\text { Duration of } \\
\text { symptoms } \\
\text { (months) }\end{array}$ & $\begin{array}{c}\text { VA pre-operative } \\
\log M A R\end{array}$ & $\begin{array}{c}\text { VA } 6 \text { months } \\
\text { post-operative } \\
\log M A R\end{array}$ & $\begin{array}{l}\text { Hole size } \\
\qquad(\mu n)\end{array}$ & Gas & Posture & Phako & $\begin{array}{c}\text { Clinical } \\
\text { information }\end{array}$ \\
\hline 1 & 70 & $\mathrm{M}$ & $\mathrm{R}$ & 7 & 0.6 & $0.4+$ & $<500$ & C3F8 & None & No & \\
\hline 2 & 47 & $\mathrm{M}$ & $\mathrm{L}$ & 8 & 0.4 & $0.3+$ & $<500$ & SF6 & Prone & No & High myope \\
\hline 3 & 68 & $\mathrm{~F}$ & $\mathrm{R}$ & 12 & 1.0 & 0.6 & $<500$ & C3F8 & Prone & Yes & \\
\hline 4 & 54 & $\mathrm{~F}$ & $\mathrm{R}$ & 4 & 0.7 & 0.2 & $<500$ & C3F8 & None & Yes & Post RD \\
\hline 5 & 66 & $\mathrm{M}$ & $\mathrm{R}$ & 6 & 0.6 & 0.2 & $>500$ & C3F8 & None & Yes & \\
\hline 6 & 72 & $\mathrm{~F}$ & $\mathrm{R}$ & 5 & 0.5 & 0.2 & $<500$ & C3F8 & Upright & Yes & \\
\hline 7 & 75 & $\mathrm{M}$ & $\mathrm{R}$ & $>38$ & 1.0 & 0.8 & $>500$ & C3F8 & Upright & Yes & Diabetic \\
\hline 8 & 67 & $\mathrm{M}$ & $\mathrm{L}$ & 4 & 0.8 & 0.5 & $>500$ & C3F8 & None & No & \\
\hline 9 & 78 & $\mathrm{~F}$ & $\mathrm{R}$ & $>36$ & 0.9 & 0.8 & $<500$ & C3F8 & Prone & Yes & \\
\hline 10 & 56 & $\mathrm{~F}$ & $\mathrm{R}$ & $>12$ & 0.8 & 0.1 & $<500$ & C3F8 & Prone & Yes & High myope \\
\hline 11 & 68 & $\mathrm{~F}$ & $\mathrm{R}$ & 12 & 0.8 & 0.3 & $<500$ & C3F8 & Prone & Yes & $\begin{array}{l}\text { Failed first surgery, } \\
\text { redo with ICG }\end{array}$ \\
\hline 13 & 70 & $\mathrm{M}$ & $\mathrm{L}$ & 10 & 0.4 & 0.2 & $<500$ & C3F8 & Prone & Yes & \\
\hline 14 & 64 & $\mathrm{~F}$ & $\mathrm{R}$ & 6 & 0.7 & 0.0 & $<500$ & C3F8 & Prone & Yes & \\
\hline 15 & 76 & $\mathrm{~F}$ & $\mathrm{R}$ & 10 & $1.0-$ & 0.9 & $>500$ & C3F8 & Prone & No & \\
\hline 16 & 86 & $\mathrm{~F}$ & $\mathrm{R}$ & 14 & $1.0-$ & 1.0 & $<500$ & C3F8 & Prone & Yes & Adherent vitreous \\
\hline 17 & 58 & $\mathrm{M}$ & $\mathrm{L}$ & $>14$ & 0.9 & 0.4 & $>500$ & C3F8 & Prone & Yes & \\
\hline 18 & 55 & $\mathrm{M}$ & $\mathrm{R}$ & 13 & 0.6 & 0.4 & $<500$ & C3F8 & Prone & Yes & \\
\hline 19 & 75 & $\mathrm{M}$ & $\mathrm{R}$ & 3 & 0.6 & 0.2 & $<500$ & C3F8 & None & Yes & \\
\hline 20 & 75 & $\mathrm{M}$ & $\mathrm{R}$ & $>6$ & $1.0-$ & 0.8 & $>500$ & C3F8 & None & Yes & \\
\hline
\end{tabular}

One patient had an open hole after the first surgery and was re-operated using ICG to confirm the complete removal of the ILM. Indeed, no remaining ILM was found with ICG and the hole closed after the second surgery. Nineteen patients had C3F8 as the intraocular tamponade agent of choice and one patient had SF6. Twelve patients postured face down for 5 days, including the patient that failed. Eight patients were not instructed to posture, but were asked to not sleep supine.

Post-operative BCVA in logMAR units varied from 1.0 to 0.0 at 6 months (mean 0.35 , median 0.3 ).

BCVA was improved or maintained in all patients. Sixteen patients $(80 \%)$ improved two or more lines of vision. All patients were followed for a minimum of 6 months. During follow-up, no patient had a retinal detachment, one patient had macular pigment alterations, but no vision loss, and there was no case of endophthalmitis.

Table 1 gives an overview of patient demographics and results.

\section{Discussion}

Macular hole surgery is frequently performed by vitreoretinal surgeons. Dyes are often used to stain the ILM with or without an epiretinal component as it facilitates a more complete and controlled removal of ILM and ERM leading to a higher primary success rate, especially in larger, stage 4 holes. ${ }^{12}$ However, the dye must be safe, and preferably easy to apply, using simple steps.

ICG has been used for both ERM and macular hole surgery. It stains the ILM intensely, thereby facilitating surgery. ${ }^{12,13}$ However, its use is not without risk. Several case reports and case series document adverse effects from a reduced visual recovery after surgery to the development of pigment alterations, ${ }^{14}$ and post-operative vision loss, ${ }^{15,16}$ particularly when retinal integrity is compromised. ${ }^{17}$ In vitro as well as in vivo toxicity has been shown against the retinal pigment epithelium and retinal glia. ${ }^{13,14,16-23}$ Owing to the risks associated with the use of ICG, alternative dyes have been sought to achieve the same goal, but with less potential toxicity.

Trypan blue was initially used to stain the lens capsule (0.06\% Trypan blue in VisionBlue, DORC, Zuidland, The Netherlands). ${ }^{24}$ At a higher concentration, Trypan blue is capable of staining preretinal structures (MembraneBlue (DORC) $0.15 \%) .{ }^{4}$ As compared with ICG, Trypan blue appears to have a broader safety profile, as it is non-toxic to cultured retinal pigment epithelial cells and glial cells at concentrations used clinically between 0.06 and $0.2 \%$, and is non-toxic to rabbit retina when it is promptly removed. ${ }^{18,23,25-27}$. Compared with ICG, it is easier to remove from the vitreous cavity, given its high water solubility. In addition, it does not appear to get incorporated into or adherent to RPE cells as much as ICG. ${ }^{20,28}$ Osmolality and $\mathrm{pH}$ of the Trypan blue solution 
applied to the retinal surface was in a physiologic range: $320 \mathrm{mOsm}$ and $\mathrm{pH} 7.0$ on repeat determinations by the hospital pharmacy and in our laboratory. Osmolality and $\mathrm{pH}$ vary depending on the buffer used. ${ }^{25,29}$ Costa et $a l^{29}$ examined the osmolarity of a number of intraocular dyes prepared in several diluents. They found that the osmolality of Trypan blue 0.5 and $0.05 \%$ mixed with BSS or glucose 5\% ranged from 287 to $332 \mathrm{mOsm}$, and the $\mathrm{pH}$ varied from 6.12 to 7.84 . The concentration of commercially available MembraneBlue (DORC) is $0.15 \%$. Variations in $\mathrm{pH}$ for ICG were found to be much more significant and potentially detrimental to the retina.

Trypan blue has been used for ERM peeling and ILM peeling in concentrations varying from 0.06 to $0.2 \% .^{5,6,8-11,29-31}$ None of these concentrations were reported as toxic in clinical practice. Haritoglou et al ${ }^{5}$ found visions 6 months after ERM removal to be similar between eyes operated with and without Trypan blue. Balayre et $a l^{32}$ operated on patients with ERMs using $0.15 \%$ Trypan blue. They then performed multifocal ERG on patients and found no decrease in macular response and an increase 4 months after surgery. Anatomical evaluation shows that there was no increase in apoptosis or other indicators of retinal toxicity when HTB was used. . $^{52,33}$

The main disadvantage of Trypan blue, as formulated in MembraneBlue (DORC), is that an air-fluid exchange (AFX) is required to achieve adequate staining. The procedure itself increases the risk of surgical complications including retinal tears. ${ }^{5,22}$ It also makes repeat applications of the dye less practical. To eliminate the need for an AFX, it is possible to either mix the dye with glucose to increase the osmolarity or to decrease the osmolarity by changing the infusion fluid (eg to Hartmann solution). We proposed a heavy form of Trypan blue, which can be applied into a fluid-filled eye without dispersing. The 1:1 mixture of MembraneBlue with $10 \%$ glucose gives staining characteristics that are sufficient to initiate and carry out an ILM peel. The staining is even more intense and visible by repeating the dye application once the ILM peel has been initiated. By eliminating the need for a fluid-air exchange, repeat application of the dye can be performed rapidly and safely. Ninety five per cent of our patients had a closed macular hole after the first surgery.

We would like to conclude that Trypan blue rendered 'heavy' by mixing it isovolumetrically with glucose $10 \%$ seems to be a relatively safe alternative for staining ILM in macular hole surgery. It eliminates the need for an AFX and can be easily reapplied when visualisation needs to be enhanced or the edge of the ILM is lost. In our small pilot study, none of our patients lost vision and $80 \%$ improved $>2$ lines at 6 months.
A larger study will be required to confirm the safety of heavy Trypan blue.

\section{Summary}

\section{Conflict of interest}

The authors declare no conflict of interest.

\section{Acknowledgements}

We certify that all applicable institutional and governmental regulations concerning the ethical use of human volunteers were followed during this research. Appropriate approval from the hospital medical ethics committee was obtained as dictated by Dutch law for this type of medical investigation. Appropriate consent was obtained.

\section{References}

1 Furino C, Micelli FT, Boscia F, Cardascia N, Recchimurzo N, Sborgia C. Triamcinolone-assisted pars plana vitrectomy for proliferative vitreoretinopathy. Retina 2003; 23: 771-776.

2 Enaida H, Hisatomi T, Hata Y, Ueno A, Goto Y, Yamada T et al. Brilliant blue $\mathrm{G}$ selectively stains the internal limiting membrane/brilliant blue G-assisted membrane peeling. Retina 2006; 26: 631-636.

3 Enaida H, Hisatomi T, Goto Y, Hata Y, Ueno A, Miura M et al. Preclinical investigation of internal limiting membrane staining and peeling using intravitreal brilliant blue G. Retina 2006; 26: 623-630.

4 Feron EJ, Veckeneer M, Parys-Van GR, Van LA, Melles GR, Stalmans P. Trypan blue staining of epiretinal membranes in proliferative vitreoretinopathy. Arch Ophthalmol 2002; 120: 141-144.

5 Haritoglou C, Eibl K, Schaumberger M, Mueller AJ, Priglinger S, Alge $\mathrm{C}$ et al. Functional outcome after Trypan blue-assisted vitrectomy for macular pucker: a prospective, randomized, comparative trial. Am J Ophthalmol 2004; 138: $1-5$.

6 Perrier M, Sebag M. Trypan blue-assisted peeling of the internal limiting membrane during macular hole surgery. Am J Ophthalmol 2003; 135: 903-905. 
7 Teba FA, Mohr A, Eckardt C, Wong D, Kusaka S, Joondeph BC et al. Trypan blue staining in vitreoretinal surgery. Ophthalmology 2003; 110: 2409-2412.

8 Vote BJ, Russell MK, Joondeph BC. Trypan blue-assisted vitrectomy. Retina 2004; 24: 736-738.

9 Hasler PW, Prunte C. Early foveal recovery after macular hole surgery. Br J Ophthalmol 2008; 92: 645-649.

10 Michalewska Z, Michalewski J, Cisiecki S, Adelman R, Nawrocki J. Correlation between foveal structure and visual outcome following macular hole surgery: a spectral optical coherence tomography study. Graefes Arch Clin Exp Ophthalmol 2008; 246: 823-830.

11 Lesnik Oberstein SY, Mura M, Tan SH, de S. Heavy Trypan blue staining of epiretinal membranes: an alternative to infracyanine green. Br J Ophthalmol 2007; 91: 955-957.

12 Bainbridge J, Herbert E, Gregor Z. Macular holes: vitreoretinal relationships and surgical approaches. Eye 2008; 22: 1301-1309.

13 Gandorfer A, Messmer EM, Ulbig MW, Kampik A. Indocyanine green selectively stains the internal limiting membrane. Am J Ophthalmol 2001; 131: 387-388.

14 Engelbrecht NE, Freeman J, Sternberg Jr P, Aaberg Sr TM, Aaberg Jr TM, Martin DF et al. Retinal pigment epithelial changes after macular hole surgery with indocyanine green-assisted internal limiting membrane peeling. Am J Ophthalmol 2002; 133: 89-94.

15 Haritoglou C, Gass CA, Schaumberger M, Ehrt O, Gandorfer A, Kampik A. Macular changes after peeling of the internal limiting membrane in macular hole surgery. Am J Ophthalmol 2001; 132: 363-368.

16 Weinberger AW, Kirchhof B, Mazinani BE, Schrage NF. Persistent indocyanine green (ICG) fluorescence 6 weeks after intraocular ICG administration for macular hole surgery. Graefes Arch Clin Exp Ophthalmol 2001; 239: 388-390.

17 Gandorfer A, Haritoglou C, Gass CA, Ulbig MW, Kampik A. Indocyanine green-assisted peeling of the internal limiting membrane may cause retinal damage. Am J Ophthalmol 2001; 132: 431-433.

18 Gale JS, Proulx AA, Gonder JR, Mao AJ, Hutnik CM. Comparison of the in vitro toxicity of indocyanine green to that of Trypan blue in human retinal pigment epithelium cell cultures. Am J Ophthalmol 2004; 138: 64-69.

19 Haritoglou C, Gandorfer A, Gass CA, Kampik A. Histology of the vitreoretinal interface after staining of the internal limiting membrane using glucose $5 \%$ diluted indocyanine and infracyanine green. Am J Ophthalmol 2004; 137: 345-348.

20 Hirasawa H, Yanagi Y, Tamaki Y, Inoue Y, Kadonosono K. Indocyanine green and Trypan blue: intracellular uptake and extracellular binding by human retinal pigment epithelial cells. Retina 2007; 27: 375-378.
21 Jackson TL, Vote B, Knight BC, El-Amir A, Stanford MR, Marshall J. Safety testing of infracyanine green using retinal pigment epithelium and glial cell cultures. Invest Ophthalmol Vis Sci 2004; 45: 3697-3703.

22 Kwok AK, Lai TY, Li WW, Yew DT, Wong VW. Trypan blue- and indocyanine green-assisted epiretinal membrane surgery: clinical and histopathological studies. Eye 2004; 18: 882-888.

23 Kwok AK, Yeung CK, Lai TY, Chan KP, Pang CP. Effects of Trypan blue on cell viability and gene expression in human retinal pigment epithelial cells. Br J Ophthalmol 2004; 88: 1590-1594.

24 Melles GR, de Waard PW, Pameyer JH, Houdijn BW. Trypan blue capsule staining to visualize the capsulorhexis in cataract surgery. J Cataract Refract Surg 1999; 25: 7-9.

25 Penha FM, Maia M, Eid FM, Príncipe AH, Freymüller EH, Maia A et al. Effects of subretinal injections of indocyanine green, Trypan blue, and glucose in rabbit eyes. Ophthalmology 2007; 114: 899-908.

26 Veckeneer M, Van OK, Monzer J, Kobuch K, van Marle W, Spekreijse $\mathrm{H}$ et al. Ocular toxicity study of Trypan blue injected into the vitreous cavity of rabbit eyes. Graefes Arch Clin Exp Ophthalmol 2001; 239: 698-704.

27 Stalmans P, Van Aken EH, Melles G, Veckeneer M, Feron EJ, Stalmans I. Trypan blue not toxic for retinal pigment epithelium in vitro. Am J Ophthalmol 2003; 135: 234-236.

28 Jin Y, Uchida S, Yanagi Y, Aihara M, Araie M. Neurotoxic effects of Trypan blue on rat retinal ganglion cells. Exp Eye Res 2005; 81(4): 395-400.

29 Costa EP, Rodrigues EB, Farah ME, Dib E, Penha F, Magalhães Jr O. et al. Vital dyes and light sources for chromovitrectomy: comparative assessment of osmolarity, $\mathrm{pH}$, and spectrophotometry. Invest Ophthalmol Vis Sci 2009; 50: $385-391$.

30 Li K, Wong D, Hiscott P, Stanga P, Groenewald C, McGalliard J. Trypan blue staining of internal limiting membrane and epiretinal membrane during vitrectomy: visual results and histopathological findings. $\mathrm{Br} \mathrm{J}$ Ophthalmol 2003; 87: 216-219.

31 Perrier M, Sebag M. Epiretinal membrane surgery assisted by Trypan blue. Am J Ophthalmol 2003; 135: 909-911.

32 Balayre S, Boissonnot M, Paquereau J, Dighiero P. [Evaluation of Trypan blue toxicity in idiopathic epiretinal membrane surgery with macular function test using multifocal electroretinography: seven prospective case studies]. J Fr Ophtalmol 2005; 28: 169-176.

33 Balayre S, Boissonnot M, Fernandez B, Quellard N, Babin P, Dighiero P. [Ultrastructural study of epiretinal membrane stained by Trypan blue: 15 case reports]. J Fr Ophtalmol 2005; 28: 159-167. 